\title{
Tie Dyeing Patten Simulation Design Based on Processing Language
}

\author{
Chendi Zhu ${ }^{1}$ Jianxia Su ${ }^{1, a}$ \\ ${ }^{1}$ Information Engineering school of Beijing Institute Of Fashion Technology \\ gxysujx@bift.edu.cn
}

Key words: tie dyeing, pattern, simulation

Abstract. This paper mainly discusses how to use computer language Processing to simulate the patterns of traditional tie dyeing pattern, and strive to maximize the reduction of the tie dye and other paintings by the formation of the pattern effects. The results prove that the effects are good, and the computer simulation technology can replace the traditional manual technology to save the manpower and material resources.

\section{Introduction}

Traditional tie dyeing methods refers to with a needle and thread woven fabric take sewing, hanging, clamping, bags and other techniques, the stamp, which has special effect. Manual tie dyeing requires many operations after seam, twisting and package, winding, folding, clip set of multi process to produce exquisite realistic graphics. The simulations of traditional tie dyeing pattern become more and more important. Processing is a new computer language, which is an extension of Java language. In modern computer languages, any advanced computer language that supports the graphical interface can be realized by the algorithm, graphics library and hardware support to achieve interactive programming.

Processing language can efficiently design graphics images with rich color and expressive force, and it does not require complicated grammar, which is determined by its own characteristics.

\section{Tie dyeing patterns design}

Extensive source of tie dyeing patterns, rich topics, including plants and flowers, birds and fish, geometric patterns and so on, traditional labor people love a variety of patterns to decorate the daily life of accessories, dress is no exception. The characteristics of tie dyeing technology determine the patterns in the form of a single pattern, sequential patterns, symmetric patterns and integrative pattern.

Method and process of traditional tie dye determines the effect of tie dyeing and expressive force, traditional techniques: tying method, folding ligation, twisted seam method, winding needle cutter gap method, clip ligation method, integrated a variety of tie method: the bundle, comprehensive application of twisted seam and plywood and so on a variety of techniques, different groups together colorful tie dyeing effect can be obtained.

\section{Continuous pattern rendering}

Processing programming can be used in the translation, rotation and scaling functions to enhance the expression of a single pattern, so that the screen has a stronger impact. Figure 1 is using exponential f unction $\mathrm{y}=\mathrm{x} 4$ and $\mathrm{y}=1-(1-\mathrm{x}) 4$ and the formation of a flower pattern, Figure 2 use rotation function rotate the pattern around the corner respectively rotating pi / 2, PI, and 3/2*PI 2pi initially formed the pattern of a flower, use the zoom function, scale can be will a single flower 
pattern around the corner to around amplification, similar to tie dye folding ligation, the pattern itself that is the symmetrical structure, as shown in figure 2. $\mathrm{r}$ The rotation of the two square continuous patterns is showed in figure 3. Procedures for the use of a stack function to pushmatrix() and function popmatrix() to a separate petals bundled into to center as the origin to the right-hand side, bottom, right below the translation of individual, respectively using the translate function, set in advance good corresponding variables, will be able to spread to expected patterns. The effect of figure 4 is obtained.

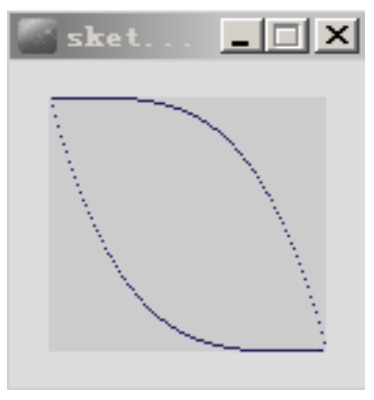

Fig.1 A leaf

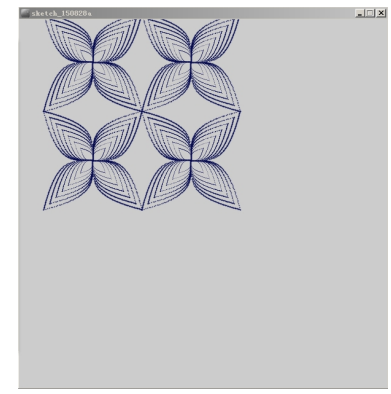

Fig.3 Symmetric pattern

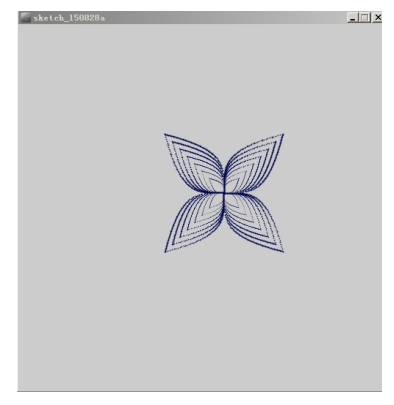

Fig.2 A flower

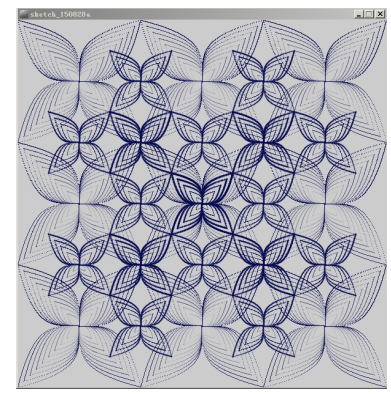

Fig.4 Two sides pattern.

\section{The drawing of continuous rotating pattern}

Computer rendering the snowflake process is not complicated, first on the coordinates of the paper depicts a snowflake, then mark each point of the coordinates, using multiple vertex function vertex () implementation, as shown below, and then move to the origin, the use of functions and the use of the function of the six sides of the shape, then the entire hexagonal snowflakes tied together into a function of the scale to achieve three snowflakes, and put together the two functions of the binding function call spin, programming using fill (0) function to get the bottom plate as shown in figure 6 .
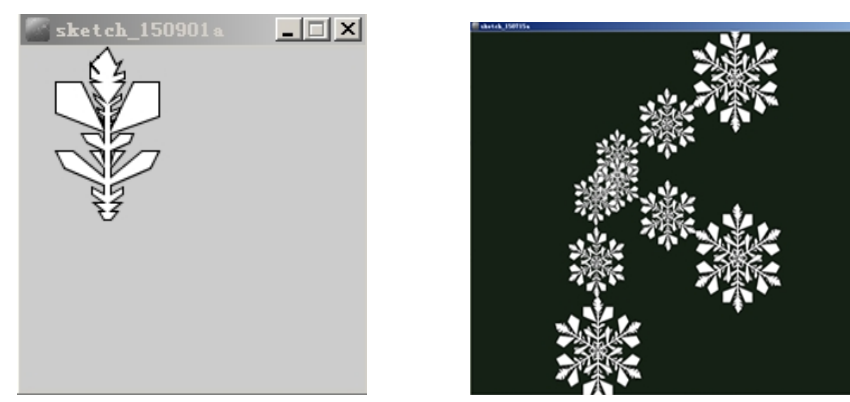

Fig.5 a snowflake

Fig.6 snowflake pattern Integrated pattern drawing

In tie dyeing technology, the use of a variety of skills will form different patterns on a piece of textile, this will make the effect more prominent, and by Processing, with sub module 
programming, each part will show different patterns, shapes of color pattern of fusion effect will match each other, such as Figure 7 and 8.

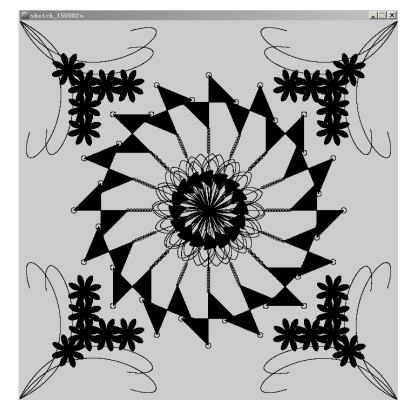

Fig.7 Combined pattern 1

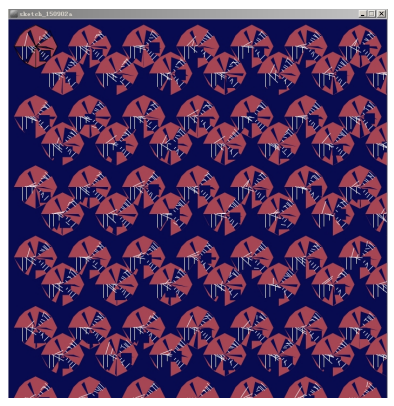

Fig.8 Combined pattern 2

\section{Random functions and tie dyeing color}

Function random is used to obtain a random value within the range of parameters,

In some cases, some of the programs are not expected to be used, but the random values are the same, the randomSeed (value) is used to generate the random numbers. Noise function is a more controllable method to generate random numbers, it is the difference between the number of operations to generate more smooth random number. Noise function has one to three parameters: noise functions can generate a single sequence of random numbers, and increase the parameters can generate random noise in more dimensions. The noise function with two parameters can generate a two-dimensional texture, with three parameters of the noise function can be used to generate three-dimensional shape or texture, or a dynamic two-dimensional texture.

\section{Application examples of random functions}

The circular patterns and flower shaped pattern package into two functions, figure 9 the translation of coordinate transformation, and size are used as variables to call, call set translate (random $(0$, width), random $(0$, height), scale (random $(0.1,1)$. It means that translation is in the range of the window and zoom scale is original 0.1 to 1 times, each run of results are not all the same. And in Figure 10 in the pattern of filling RGB as a variable called, each pattern of filling RGB are not all the same, run after the effect of more colorful.
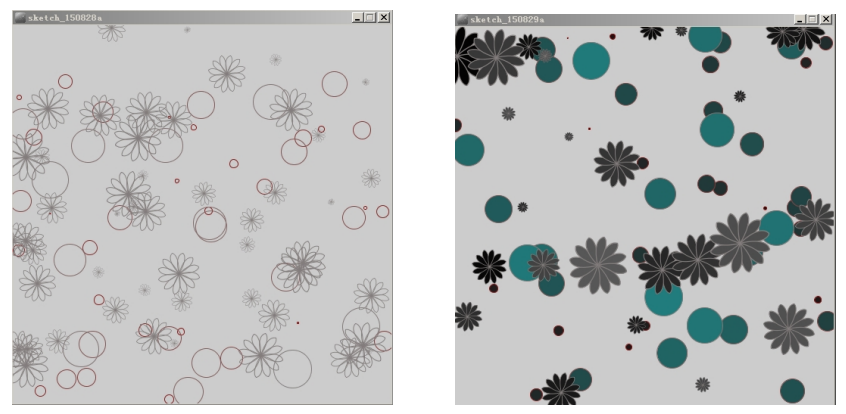

Fig.9 Random factor1 Fig.10 Random factor2 Random function rendering gradient

Tie dyeing process is to aft 'er bundling techniques basic fabric, color fabric immersed in liquid. Commonly used staining techniques have dip method; boiling dye method; steam dyeing method.

The dye molecules immersed in different parts of each fabric quantity also means that each pixel is different and the pixel changes irregularly. So as long as the random function of the variable factor into pixels can be. As shown below. 


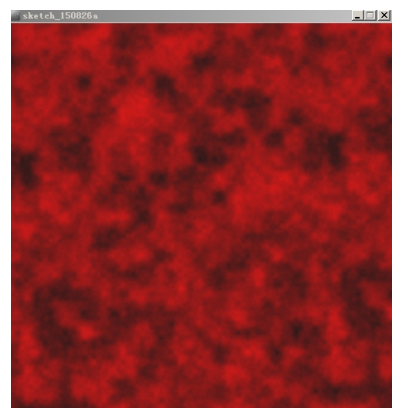

Fig.11 random factor1

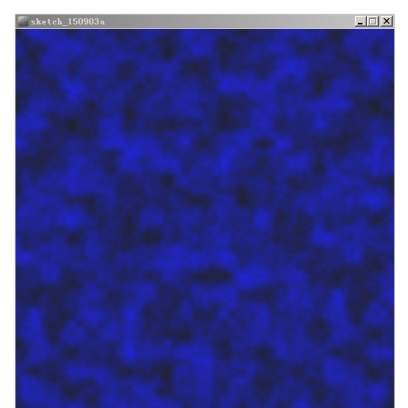

Fig.12 random factor2

\section{Gradient pattern}

Many of the tie dyeing patterns are formed by the gradual, as mentioned previously, gradient is to control each pixel of pattern to make one point with a bit of a micro cents change, the naked eye can not easily observed it. And when the dot is condensed into a pattern, these tiny changes will be revealed, for the following illustration, Figure 13,14 are gradient color circles.

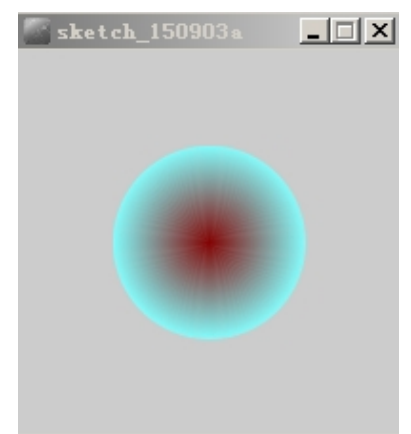

Fig.13 gradient circle1

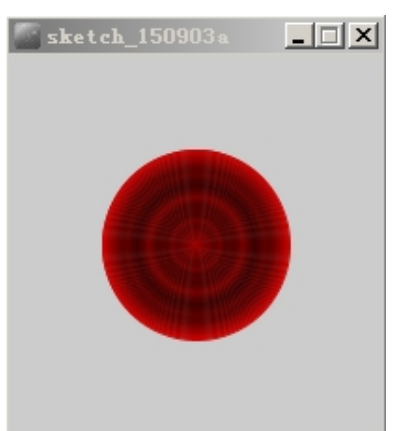

Fig.14 gradient circle2

Add ordered patterns in the original two-dimensional gradient background is formed as shown in the results. Using the Bessel function to change the parameters can come out an arc type of rose petal pattern, so that the origin of the circle around the origin of a circle, and so magnified 1.3 times 6 times, using the ideal position of the translation function. The program is encapsulated into an independent function, and the translation function is to be used as a variable, such as a rule, and the disordered pattern is created.

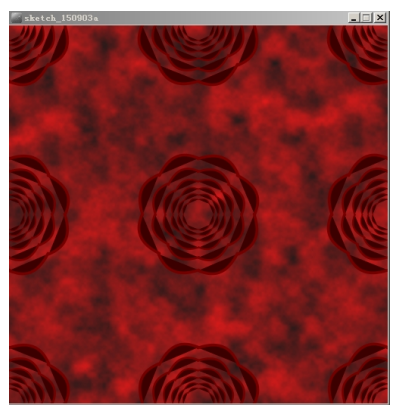

Fig.15. The disordered pattern

\section{Summary}

This paper realized the design and simulation of several traditional tie dyeing patterns based on processing language. Some programming methods are discussed in detail. The simulation patterns shows that program simulations are useful. But the simulation of traditional tie dying pattern is still long way to go. 


\section{Acknowledgments}

This paper is sponsored by KYJH02140201/025 of 2014 project of Beijing Science and Technology.

\section{Reference}

[1] The definitive guide to processing language (USA) Rees, Foley with; Zhang Jing, et al. Beijing: Publishing House of electronics industry, 2013.10

[2] Modern tie dyeing art characteristics Li Hui, Chen Mingzhen; Wuhan University of Science and Engineering; TS193.53

[3] Computer aided design tie dye graphics Wang Shuying, Hong Fu Wang; Jiangnan University, College of textile and garment; 1673-0356 (2009) 04-0048-02

[4] The pattern of tie dyeing and its characteristics of making Jiang Yuping Nantong Vocational College

[5] Application of fractal patterns in fashion design [D]. Beijing: Beijing Institute Of Fashion Technology, 2010 